PROCEEDINGS OF THE

AMERICAN MATHEMATICAL SOCIETY

Volume 125, Number 7, July 1997, Pages 2033-2039

S 0002-9939(97)03833-1

\title{
INNER DERIVATIONS ON ULTRAPRIME NORMED ALGEBRAS
}

\author{
M. CABRERA AND J. MARTÍNEZ
}

(Communicated by Palle E. T. Jorgensen)

\begin{abstract}
We show that, for every ultraprime Banach algebra $A$, there exists a positive number $\gamma$ satisfying $\gamma\|a+Z(A)\| \leq\left\|D_{a}\right\|$ for all $a$ in $A$, where $Z(A)$ denotes the centre of $A$ and $D_{a}$ denotes the inner derivation on $A$ induced by $a$. Moreover, the number $\gamma$ depends only on the "constant of ultraprimeness" of $A$.
\end{abstract}

\section{INTRODUCTION}

Ultraprime normed associative algebras were introduced by M. Mathieu in [6] as those normed associative complex algebras having a prime ultrapower with respect to a countably incomplete ultrafilter. There these algebras were intrinsically characterized by the existence of a positive number $K$ such that

$$
K\|a\|\|b\| \leq\left\|M_{a, b}\right\|
$$

for all $a, b$ in the algebra, where $M_{a, b}$ denotes the linear operator defined by $M_{a, b}(x)=a x b$. Algebras of this type have received considerable attention in the last years, undoubtedly justified by the simplicity and elegance of the characterization given by (1) and by the fact that relevant classes of Banach algebras (for example, prime $C^{*}$-algebras [7, Proposition 2.3] and primitive Banach algebras with non-zero socle and minimum topology [1]) are ultraprime.

A classical problem in $C^{*}$-algebras is the existence and determination of a positive constant $\gamma$ such that for all $A$ in a prefixed class of algebras the inequality

$$
\gamma\|a+Z(A)\| \leq\left\|D_{a}\right\|
$$

holds for all $a$ in $A$, where $D_{a}$ is the inner derivation $x \rightarrow[a, x]=a x-x a$ and $\|a+Z(A)\|$ is the distance from $a$ to the centre $Z(A)$ of $A$ (see, for example, [10] and $[9])$. As is well known, the fact that for a given Banach algebra there exists a positive constant $\gamma$ satisfying (2) is equivalent to the fact that the set of all inner derivations of $A$ is closed within all bounded derivations on $A$. J. G. Stampfli [11] showed that $\gamma=2$ (the maximum possible value) when $A$ is a unital primitive $C^{*}$-algebra, and in particular when $A$ is the algebra of all bounded linear operators on a Hilbert space. This result has been recently extended to unital prime $C^{*}$-algebras (see [3, Proposition 2.23] or [10, Corollary 2.9]). For Banach algebras of all bounded linear operators on a Banach space B. E. Johnson [4] showed that $\gamma=2$ is not always

Received by the editors September 26, 1995 and, in revised form, January 24, 1996.

1991 Mathematics Subject Classification. Primary 47B47; Secondary 47B48, 46H05.

(C)1997 American Mathematical Society 
possible, and J. Kyle [5] showed that the equality $\gamma=2$ characterizes Hilbert spaces among all uniformly convex Banach spaces. The main result (Theorem 2) in this paper solves the existence problem of a positive constant $\gamma$ for ultraprime Banach algebras, thus extending the aforementioned result for unital prime $C^{*}$-algebras. More precisely the following is shown: To every constant $K>0$ there exists a constant $\gamma>0$ such that (2) is satisfied in every ultraprime normed algebra $A$ with constant of ultraprimeness greater than or equal to $K$ provided $A$ has a unit or its norm is complete. The techniques used to obtain this result involve ultraproducts as well as the fact that the unitization of every ultraprime Banach algebra without unit is still ultraprime for the $l_{1}$-norm (Theorem 1 ). Section 1 is devoted to proving this result. The given proof does not involve ultraproducts and gives a bound for the constant of ultraprimeness of the unitization algebra. In Section 3 we give an example showing that the assumption of completeness in Theorem 1 as well as the assumption of either existence of a unit or completeness in Theorem 2 cannot be removed.

\section{The Unitization of AN ULtraprime NORMED ALGEBRA}

In [6] M. Mathieu introduced ultraprime normed algebras and gave an equivalent characterization without any reference to ultrapowers in the following way: A normed associative complex algebra $A$ is ultraprime if there exists a constant $K>0$ such that $K\|a\|\|b\| \leq\left\|M_{a, b}\right\|$ holds for all $a, b$ in $A$, where $M_{a, b}$ denotes the two-sided multiplication operator on $A$, that is $M_{a, b}(x):=a x b$ for all $x$ in $A$. The largest possible number $K$ in the above inequality will be called the constant of ultraprimeness of $A$ and denoted by $K_{A}$. As a consequence of the theory of the normed symmetric Martindale algebra of quotients of an ultraprime normed algebra as developed in [8] and taking into account some improvement in the direction stated in [2, Theorem 1.4] we can state the following

Proposition 1. Let $(A,\|\cdot\|)$ be an ultraprime normed algebra without unit. Then the norm $\|\cdot\|$ can be extended to a unital algebra norm $|\cdot|$ on the unitization $A_{1}$ of $A$ in such a way that also $\left(A_{1},|\cdot|\right)$ is ultraprime. More precisely $K_{A_{1}} \geq K_{A}^{5}$.

Our next goal is to prove an analogous result of the above proposition in the complete case where the unitization $A_{1}$ of $A$ carries the $l_{1}$-norm (the largest unital algebra norm extending the given norm on $A$ ). Later we will show that the assumption of completeness cannot be removed, which in addition reveals an oversight in the formulation of [6, Proposition 3.7]. To realize this one has to note that the completion of a non-unital (ultraprime) normed algebra can be a unital (ultraprime) Banach algebra, the unitization of which cannot be ultraprime. The following proposition states a purely algebraic result, which perhaps is well-known. As usual, for an element $a$ in an algebra $A, L_{a}$ and $R_{a}$ will denote respectively the operators of left and right multiplication by $a$ on $A$.

Proposition 2. Let $A$ be an associative algebra. If there exists an element a in $A$ such that $L_{a}$ and $R_{a}$ are invertible in the algebra $L(A)$ of all linear operators on $A$, then $A$ has unit and $a$ is invertible in $A$.

Proof. Assume that $L_{a}$ and $R_{a}$ are invertible operators in $A$. Then there are elements $e$ and $f$ in $A$ such that $a e=a=f a$. Also, for every $x$ in $A$ there are $y$ and $z$ in $A$ such that $a y=x=z a$, and so we have $x e=z a e=z a=x$ and $f x=f a y=a y=x$. Thus, $e$ is a right unit and $f$ is a left unit for $A$; hence 
$e=f e=f$ and $A$ has unit. Changing $x$ by $e$ in the above argument we obtain that $a$ is invertible.

Theorem 1. Let $A$ be an ultraprime Banach algebra without unit. Then the unitization algebra $A_{1}$ of $A$ is an ultraprime Banach algebra for the $l_{1}$-norm. More precisely, $K_{A_{1}} \geq K_{A}^{5}\left(1+2 K_{A}\right)^{-2}$ holds for the respective constants of ultraprimeness.

Proof. First we prove that

$$
\frac{K^{2}}{1+2 K}(1+\|a\|) \leq\left\|I-L_{a}\right\|
$$

for all $a$ in $A$ and $K=K_{A}$. For every $a$ in $A$, by [8, Lemma 2.3] applied to the bounded totally defined double centralizer $\left(A, I-L_{a}, I-R_{a}\right)$, we obtain $K\left\|I-R_{a}\right\| \leq\left\|I-L_{a}\right\|$. Hence if $\left\|I-L_{a}\right\|<K$, then $\left\|I-R_{a}\right\|<1$ and $\left\|I-L_{a}\right\|<1$. Now, since in every Banach algebra with unit the open ball with radius 1 about this unit consists of invertible elements, it follows: if $\left\|I-L_{a}\right\|<K$, then $L_{a}$ and $R_{a}$ are invertible elements in the Banach algebra $B L(A)$ of all bounded linear operators on $A$, therefore they are invertible elements in the algebra $L(A)$, and by Proposition $2 A$ has a unit, which is a contradiction. Therefore

$$
K \leq\left\|I-L_{a}\right\| .
$$

On the other hand, from the definition of ultraprimeness it follows easily that

$$
K\|a\| \leq\left\|L_{a}\right\| .
$$

Adding (3) and (4) we obtain

$$
K(1+\|a\|) \leq\left\|I-L_{a}\right\|+\left\|L_{a}\right\|,
$$

and taking into account

$$
\begin{aligned}
\left\|L_{a}\right\| & =\left\|I-\left(I-L_{a}\right)\right\| \leq\left\|I-L_{a}\right\|+\|I\| \\
& =\left\|I-L_{a}\right\|+\frac{1}{K} K \leq\left(1+\frac{1}{K}\right)\left\|I-L_{a}\right\|
\end{aligned}
$$

(where (3) has been used for the last inequality), it follows that

$$
K(1+\|a\|) \leq\left(2+\frac{1}{K}\right)\left\|I-L_{a}\right\|
$$

as we claimed at the beginning of the proof.

Now we carry the above information to the action on $A$ of the elements of $A_{1}$. From now on, for $a+\lambda \mathbf{1}$ in $A_{1}$ the restriction to $A$ of the operator on $A_{1}, L_{a+\lambda \mathbf{1}}$ will be denoted by $L_{a+\lambda \mathbf{1}}^{A}$. Given $a+\lambda \mathbf{1}$ in $A_{1}$ with $\lambda \neq 0$, from the equality $L_{a+\lambda \mathbf{1}}^{A}=\lambda\left(I-L_{\left(-\lambda^{-1} a\right)}\right)$ we obtain

$$
\left\|L_{a+\lambda \mathbf{1}}^{A}\right\|=|\lambda|\left\|I-L_{\left(-\lambda^{-1} a\right)}\right\| \geq|\lambda| \frac{K^{2}}{1+2 K}\left(1+\left\|-\lambda^{-1} a\right\|\right)
$$

From this we derive as a consequence of (4) that

$$
\left\|L_{a+\lambda \mathbf{1}}^{A}\right\| \geq \frac{K^{2}}{1+2 K}(|\lambda|+\|a\|)
$$

holds for all $a+\lambda \mathbf{1}$ in $A_{1}$. 
Finally we prove that $A_{1}$ is ultraprime. By the last inequality, given $a+\lambda \mathbf{1}, b+\mu \mathbf{1}$ in $A_{1}$ and $0<\varepsilon<1$ there are norm-one elements $x, y$ in $A$ such that

$$
\left\|L_{a+\lambda \mathbf{1}}^{A}(x)\right\| \geq \varepsilon \frac{K^{2}}{1+2 K}(|\lambda|+\|a\|) \quad \text { and } \quad\left\|L_{b+\mu 1}^{A}(y)\right\| \geq \varepsilon \frac{K^{2}}{1+2 K}(|\mu|+\|b\|) .
$$

Since from the equality $M_{(a+\lambda \mathbf{1}) x,(b+\mu \mathbf{1}) y}^{A}=R_{y}^{A} M_{a+\lambda \mathbf{1}, b+\mu 1} L_{x}^{A}$ it follows that

$$
\begin{aligned}
& K\|(a+\lambda \mathbf{1}) x\|\|(b+\mu \mathbf{1}) y\| \leq\left\|M_{(a+\lambda \mathbf{1}) x,(b+\mu \mathbf{1}) y}^{A}\right\| \\
& \quad \leq\left\|R_{y}^{A}\right\|\left\|M_{a+\lambda \mathbf{1}, b+\mu \mathbf{1}}\right\|\left\|L_{x}^{A}\right\| \leq\left\|M_{a+\lambda \mathbf{1}, b+\mu \mathbf{1}}\right\|,
\end{aligned}
$$

we have

$$
\begin{aligned}
\left\|M_{a+\lambda \mathbf{1}, b+\mu \mathbf{1}}\right\| & \geq K\|(a+\lambda \mathbf{1}) x\|\|(b+\mu \mathbf{1}) y\| \\
& \geq K \varepsilon^{2}\left(\frac{K^{2}}{1+2 K}\right)^{2}(|\lambda|+\|a\|)(|\mu|+\|b\|) .
\end{aligned}
$$

Passing to the limit as $\varepsilon \rightarrow 1$, we obtain the assertion.

Remark. An alternative proof of the above theorem without an estimate for the constant of ultraprimeness of the unitization can be obtained using techniques of ultrapowers via [6, Proposition 2.4] and [2, Proposition 2.6].

\section{THE MAIN RESUlT}

Given an element $a$ in an algebra $A$, the inner derivation in $A$ induced by $a$ is the linear operator $D_{a}$ on $A$ defined by $D_{a}(x):=a x-x a$ for all $x$ in $A$. If $A$ is a normed algebra it is clear that every inner derivation is continuous and a simple application of the triangle inequality shows that $\left\|D_{a}\right\| \leq 2\|a+Z(A)\|$ for all $a$ in $A$, where $Z(A)$ denotes the centre of $A$. The problems of existence and determination of a positive constant $\gamma$ such that for all $A$ in a prefixed class of normed algebras the inequality $\gamma\|a+Z(A)\| \leq\left\|D_{a}\right\|$ holds for all $a$ in $A$, have been intensively studied in the $C^{*}$-context (see, for example, [10] and [9]). In this section we solve the existence problem in ultraprime context, extending the previously known case of unital prime $C^{*}$-algebras. The techniques used involve ultraproducts, and so they do not give bounds for $\gamma$.

An ultrafilter $\mathcal{U}$ on a set $I$ is said to be countably incomplete if it is not closed under countable intersections. Given a countably incomplete ultrafilter $\mathcal{U}$ on $I$ and a family $\left(A_{i}\right)_{i \in I}$ of normed algebras, we consider the normed algebra $l_{\infty}$-sum of this family $\prod_{i \in I} A_{i}$ and the closed ideal $N_{\mathcal{U}}$ of $\prod_{i \in I} A_{i}$ given by

$$
N_{\mathcal{U}}:=\left\{\left(a_{i}\right)_{i \in I} \in \prod_{i \in I} A_{i}: \lim _{\mathcal{U}}\left\|a_{i}\right\|=0\right\} .
$$

The (normed) ultraproduct of the family $\left(A_{i}\right)_{i \in I}$ with respect to the ultrafilter $\mathcal{U}$ is defined as the quotient algebra $\left(A_{i}\right)_{\mathcal{U}}:=\prod_{i \in I} A_{i} / N_{\mathcal{U}}$. If by abuse of notation we denote by $\left(a_{i}\right)$ the canonical projection in $\left(A_{i}\right) \mathcal{U}$ of $\left(a_{i}\right) \in \prod_{i \in I} A_{i}$, then it is easy to verify that $\left\|\left(a_{i}\right)\right\|=\lim _{\mathcal{U}}\left\|a_{i}\right\|$.

Theorem 2. Let $K$ be a constant such that $0<K \leq 1$. Then

(i) There exists a constant $\alpha(K)$ with $0<\alpha(K) \leq 2$ such that for every ultraprime normed algebra $A$ with unit and with constant of ultraprimeness greater than or equal to $K$, the inequality $\alpha(K)\|a+Z(A)\| \leq\left\|D_{a}\right\|$ holds for all a in $A$. 
(ii) There exists a constant $\beta(K)$ with $0<\beta(K) \leq 2$ such that for every ultraprime Banach algebra $A$ with constant of ultraprimeness greater than or equal to $K$, the inequality $\beta(K)\|a+Z(A)\| \leq\left\|D_{a}\right\|$ holds for all $a$ in $A$.

Proof. (i) Assume that (i) is not true. Then for each natural number $n$ there is an ultraprime normed algebra $A_{n}$ with unit and with constant of ultraprimeness greater than or equal to $K$ and there is $a_{n}$ in $A_{n}$ such that

$$
\left\|D_{a_{n}}\right\|<\frac{1}{n}\left\|a_{n}+Z\left(A_{n}\right)\right\| .
$$

In addition we can suppose that $\left\|a_{n}\right\|=\left\|a_{n}+Z\left(A_{n}\right)\right\|=1$. Indeed, since (5) implies $\left\|a_{n}+Z\left(A_{n}\right)\right\| \neq 0$, replacing $a_{n}$ by $\left\|a_{n}+Z\left(A_{n}\right)\right\|^{-1} a_{n}$ we can assume $\left\|a_{n}+Z\left(A_{n}\right)\right\|=1$. Taking into account that $Z\left(A_{n}\right)=\mathbb{C} \mathbf{1}[6$, Proposition 3.4] we get the existence of a complex number $\lambda_{n}$ such that $1=\left\|a_{n}+Z\left(A_{n}\right)\right\|=\left\|a_{n}+\lambda_{n} \mathbf{1}\right\|$. The claim is obtained replacing again $a_{n}$ by $a_{n}+\lambda_{n} \mathbf{1}$.

Let $\mathcal{U}$ be a countably incomplete ultrafilter on $\mathbb{N}$ containing the Fréchet filter, and let $A$ denote the ultraprime normed algebra with unit and with constant of ultraprimeness greater than or equal to $K$ obtained via the ultraproduct of the family $\left(A_{n}\right)_{n \in \mathbb{N}}$ with respect to $\mathcal{U}$. The sequence $\left(a_{n}\right)$ is a norm-one element in $A$ such that $\left\|D_{\left(a_{n}\right)}\right\|=\lim _{\mathcal{U}}\left\|D_{a_{n}}\right\|=0$. Therefore $\left(a_{n}\right)$ is a (norm-one) element in $Z(A)=\mathbb{C} 1[6$, Proposition 3.4], and so there exists a (non-zero) complex number $\mu$ such that $\left(a_{n}\right)=\mu \mathbf{1}$. Now, we have $0=\lim _{\mathcal{U}}\left\|a_{n}-\mu \mathbf{1}\right\|$ and $\left\|a_{n}-\mu \mathbf{1}\right\| \geq$ $\left\|a_{n}+Z\left(A_{n}\right)\right\|=1$, a contradiction.

(ii) By Theorem 1 and (i), there exists a constant $\alpha\left(K^{5} /(1+2 K)^{2}\right)$ such that for every ultraprime Banach algebra $A$ without unit and with constant of ultraprimeness $K_{A} \geq K$, the inequality $\alpha\left(K^{5} /(1+2 K)^{2}\right)\left\|a+Z\left(A_{1}\right)\right\| \leq\left\|D_{a}^{1}\right\|$ holds for all $a$ in $A$, where $D_{a}^{1}$ denotes the inner derivation in $A_{1}$ induced by $a$. Since such an algebra $A$ does not have a unit we have $Z(A)=\{0\}$ and $Z\left(A_{1}\right)=\mathbb{C} \mathbf{1}$ [6, Proposition 3.4]. Therefore $\left\|a+Z\left(A_{1}\right)\right\|=\|a\|=\|a+Z(A)\|$. On the other hand, it is clear that $\left\|D_{a}^{1}\right\|=\left\|D_{a}\right\|$, and so we have $\alpha\left(K^{5} /(1+2 K)^{2}\right)\|a+Z(A)\| \leq\left\|D_{a}\right\|$ for all $a$ in $A$. Finally, for ultraprime Banach algebras with unit and constant of ultraprimeness greater than or equal to $K$, it follows from (i) that $\alpha(K)\|a+Z(A)\| \leq\left\|D_{a}\right\|$. The proof concludes taking $\beta(K):=\operatorname{Min}\left\{\alpha(K), \alpha\left(K^{5} /(1+2 K)^{2}\right)\right\}$.

\section{An exAmple}

In this section we give an example of an ultraprime non-complete normed algebra without unit whose unitization for the $l_{1}$-norm is not ultraprime. This shows that the assumption of completeness in Theorem 1 cannot be removed. The example also allows an analysis of Theorem 2, showing that the assumption of existence of a unit in the assertion (i) and the assumption of completeness in the assertion (ii) cannot be removed.

We recall that an element $a$ in an associative algebra $A$ is called algebraic if the subalgebra of $A$ generated by $a$ has finite dimension, equivalently, if there exists a non-zero polynomial $p$ with coefficients in the base field such that $p(a)=0$. It is clear that zero is the only algebraic element in the subalgebra generated by a non-algebraic element. Also note that if the algebra $A$ has a unit $\mathbf{1}$ and $a$ is an algebraic element in $A$, then $a+\alpha \mathbf{1}$ is algebraic for every scalar $\alpha$.

Proposition 3. Let $H$ be an infinite-dimensional complex Hilbert space, and let $A$ be the $\left(C^{*}-\right)$ subalgebra of $B L(H)$ generated by the identity operator $\operatorname{Id}_{H}$ and 
$K L(H)$, the ideal of all compact operators on $H$. Fix a non-algebraic operator $T$ in $K L(H)$ (for example a compact operator $T$ with infinite spectrum) and let $B$ denote the subalgebra of $A$ generated by $T+\operatorname{Id}_{H}$. If $F L(H)$ denotes the ideal of $B L(H)$ of all finite range operators, then $C:=F L(H)+B$ is a dense non-unital subalgebra of the unital prime $C^{*}$-algebra $A . C$ is an ultraprime normed algebra without unit whose unitization for the $l_{1}$-norm is not ultraprime, and there is no positive constant $\gamma$ such that $\gamma\|c+Z(C)\| \leq\left\|D_{c}\right\|$ for all $c$ in $C$.

Proof. Clearly $C$ is a subalgebra of $A$. Moreover $C$ is dense in $A$ because $F L(H)$ is dense in $K L(H)$ and if $\left\{F_{n}\right\}$ is a sequence in $F L(H)$ convergent to $T$, then $\left\{-F_{n}+\left(T+\mathrm{Id}_{H}\right)\right\}$ is a sequence in $C$ that converges to $\operatorname{Id}_{H}$. Moreover we have $\operatorname{Id}_{H} \notin C$. Indeed, if $\operatorname{Id}_{H} \in C$, then there exists $F \in F L(H)$ such that $\operatorname{Id}_{H}-F \in B$, therefore $\operatorname{Id}_{H}-F=0$ because zero is the only algebraic element in $B$, and so $\operatorname{Id}_{H}=F \in F L(H)$, a contradiction. Now, as $C$ is dense in $A$ and $\operatorname{Id}_{H}$ is the unit of $A$, it follows that $C$ cannot have a unit. By [7, Proposition 2.3] every prime $C^{*}$-algebra is ultraprime and by [6, Proposition 3.5] every dense subalgebra of an ultraprime algebra is still ultraprime, hence we have that $C$ is an ultraprime algebra. Now we will prove that the unitization algebra $C_{1}$ of $C$ is not ultraprime. Since the unitization of a prime algebra with unit is not prime, it follows that $A_{1}$ is not prime. On the other hand, the fact that $C$ is dense in $A$ yields an isometric isomorphism from the completion of $C_{1}$ onto $A_{1}$, therefore the completion of $C_{1}$ is a non-ultraprime (in fact non-prime) algebra, and so $C_{1}$ is a non-ultraprime algebra [6, Proposition 3.5]. Finally, given a sequence $\left\{T_{n}\right\}$ in $C$ that converges to $\operatorname{Id}_{H}$, we have that the sequences of multiplication operators $\left\{L_{T_{n}}\right\}$ and $\left\{R_{T_{n}}\right\}$ converge to $\operatorname{Id}_{C}$. Therefore $\left\{D_{T_{n}}\right\}=\left\{L_{T_{n}}-R_{T_{n}}\right\} \rightarrow 0$, and so there is no positive constant $\gamma$ with $\left(\gamma\left\|T_{n}\right\|=\right) \gamma\left\|T_{n}+Z(C)\right\| \leq\left\|D_{T_{n}}\right\|$ for all $n$ in $\mathbb{N}$.

\section{ACKNOWLEDGMENTS}

We would like to thank A. Rodríguez Palacios for a valuable suggestion in the search of the above example. We would also like to thank the referee for making some helpful comments about this work.

\section{REFERENCES}

1. P. Ara and M. Mathieu, On ultraprime Banach algebras with non-zero socle, Proc. Roy. Irish Acad. 91A (1991), 89-98. MR 93h:46061

2. M. Cabrera and A. Rodríguez, Non-degenerately ultraprime Jordan-Banach algebras: A Zel'manovian treatment, Proc. London Math. Soc. 69 (1994), 576-604. MR 95g:46094

3. L. A. Fialkow, Structural properties of elementary operators, in Elementary operators and applications, Proc. Int. Workshop, Blaubeuren, June 1991; World Scientific, Singapore, 1992, 55-113. MR 93i:47042

4. B. E. Johnson, Norms of derivations on $\mathcal{L}(\mathcal{X})$, Pacific J. Math. 38 (1971), 465-469. MR 46:6087

5. J. Kyle, Norms of derivations, J. London Math. Soc. 16 (1977), 297-312. MR 58:7113

6. M. Mathieu, Rings of quotients of ultraprime Banach algebras with applications to elementary operators, Proc. Centre Math. Anal. Austral. Nat. Univ. 21 (1989), 297-317. MR 91a:46054

7. _ Elementary operators on prime $C^{*}$-algebras, I. Math. Ann. 284 (1989), 223-244. MR 90h:46092

8. _ The symmetric algebra of quotients of an ultraprime Banach algebra, J. Austral. Math. Soc. Ser. A 50 (1991), 75-87. MR 92g:46061

9. and P. E. T. Jorgensen, Birkhauser, Basel-Boston, 1994, pp. 144-152. MR 95g:46128 
10. D. W. B. Somerset, Inner derivations and primal ideals of $C^{*}$-algebras, J. London Math. Soc. 50 (1994), 568-580. MR 95h:46107

11. G. J. Stampfli, The norm of a derivation, Pacific J. Math. 33 (1970), 737-747. MR 42:861

Departamento de Análisis Matemático, Facultad de Ciencias, Universidad de Granada, 18071-GRANADA, Spain

E-mail address: cabrera@goliat.ugr.es

E-mail address: jmmoreno@goliat.ugr.es 